\title{
High mobility ambipolar charge transport in a cross-linked reactive mesogen at room temperature
}

\author{
T. Kreouzis ${ }^{\text {a) }}$ and R. J. Baldwin \\ Physics Department, Queen Mary, University of London, E1 4NS, United Kingdom \\ M. Shkunov, I. McCulloch, M. Heeney, and W. Zhang \\ Merck Chemicals, Chilworth Science Park, Southampton, SO16 7QD, United Kingdom
}

(Received 20 May 2005; accepted 1 September 2005; published online 21 October 2005)

\begin{abstract}
By photochemically cross linking the molecules of a reactive end group functionalized calamitic liquid crystal (bis(heptylphenyl)-bithiophene-oxetane) in the smectic mesophase, an insoluble rigid molecular network is formed which remains structurally unchanged on cooling to room temperature. The charge transport is investigated by the time-of-flight method and the resulting material shows long-range $(\sim 10 \mu \mathrm{m})$ ambipolar transport with nondispersive field independent hole mobility, $\mu_{\text {hole }}=0.016 \mathrm{~cm}^{2} / \mathrm{V} \mathrm{s}$ and dispersive field dependent electron mobility, typically $\mu_{\text {electron }}$ $=0.028 \mathrm{~cm}^{2} / \mathrm{V} \mathrm{s}$ (both at $4 \times 10^{4} \mathrm{~V} \mathrm{~cm}^{-1}$ and at $15^{\circ} \mathrm{C}$ ). These desirable charge transport properties make this system a promising candidate for organic electronic applications, such as charge transport layers in organic light-emitting diodes and field effect transistors. (C) 2005 American Institute of Physics. [DOI: 10.1063/1.2117632]
\end{abstract}

Highly ordered thermotropic liquid crystalline mesophases display desirable high mobility charge transport; ${ }^{1}$ these usually occur at elevated temperatures, and upon cooling, the formation of a polycrystalline solid has a detrimental effect on the charge transport ${ }^{1}$ as the grain boundaries form charge carrier barriers. These barriers have the effect of reducing the carrier range and long-range mobility. It has been proposed that the problem of maintaining the enhanced transport properties present in the mesophase, at temperatures below the crystalline to mesophase transition, can be solved by the use of reactive mesogens (RMs). These are comprised of liquid crystal molecules with reactive end groups at the ends of the aliphatic chains. They form thermotropic mesophases and can be irreversibly photopolymerized in the presence of an appropriate photoinitiator, forming a stable network. It is expected that as the morphology is frozen-in, the transport properties will be stabilized. ${ }^{2,3}$ The formation of an insoluble semiconducting film has the advantage of simplifying the fabrication of multilayer devices using sequential solution-based application methods to add further layers. ${ }^{3}$ In addition, RMs can be patterned using optical masks.

Initial time-of-flight (TOF) studies on a nonreactive calamitic liquid crystal bis(alkylphenyl)-bithiophene (PTTP) (Fig. 1(a)] in the smectic phase (SmG) showed ambipolar transport [see Fig. 2) with resulting hole mobility $\mu_{\text {hole }}$ $=0.044 \mathrm{~cm}^{2} \mathrm{~V}^{-1} \mathrm{~s}^{-1}$ and electron mobility $\mu_{\text {electron }}$ $=0.07 \mathrm{~cm}^{2} \mathrm{~V}^{-1} \mathrm{~s}^{-1}$ (these are both dispersive and field dependent, quoted here at $4 \times 10^{4} \mathrm{~V} \mathrm{~cm}^{-1}$ ), indicating that this particular calamitic core was a promising candidate for the preparation of an oxetane containing reactive mesogen. Being a thermotropic liquid crystal at elevated temperatures, this behavior was, of course, lost at room temperature, yielding featureless trapping decays in the photocurrent transients.

The RM used in the cross linking experiment (PTTPoxetane) is shown in Fig. 1(b). This was mixed with 0.5

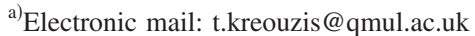

wt \% cationic initiator (Meerkat) and the liquid crystal cell (thickness, $d=10 \mu \mathrm{m}$ ) was filled by capillary action by melting the solid above the clearing point in a modified Linkam LTS350 hotstage. The RM was then allowed to form the smectic mesophase $(\mathrm{SmB})$ by lowering the temperature to $140{ }^{\circ} \mathrm{C}$ and cross-linked using the $405 \mathrm{~nm}$ wavelength output from a continuous-wave diode laser $(15 \mathrm{~mW})$ for $60 \mathrm{~min}$. The cross-linking was inferred by heating the sample above the clearing point temperature, and comparing the illuminated regions to those not illuminated under a polarized optical microscope. The cross-linked regions maintained their birefringent texture, while those that were not subject to radiation underwent the transition to the isotropic phase. Direct confirmation of the cross-linking reaction by infared spectroscopy was not possible as the sample was encased within the glass liquid crystal cell. The sample was cooled to room temperature $\left(15^{\circ} \mathrm{C}\right)$ and the TOF photocurrent transients were obtained using $6 \mathrm{~ns}$ duration, $337 \mathrm{~nm}$ wavelength, laser pulses from a Lambda Physic $\mathrm{N}_{2}$ laser, with the sample under a dc bias, magnitude $V$, whose polarity determines the sign of carrier investigated. The photocurrents were detected as the voltage droped across a load resistor on the input of an Agilent infinium digitizing oscilloscope. Figure 3 shows two typical hole photocurrents at room temperature; they show a nondispersive constant current plateau, and the inflection point transit time $\left(t_{0}\right)$ scales correctly with varying electric

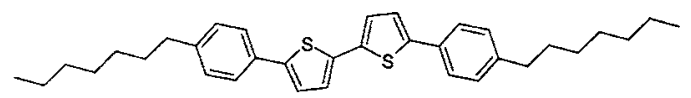

(a)

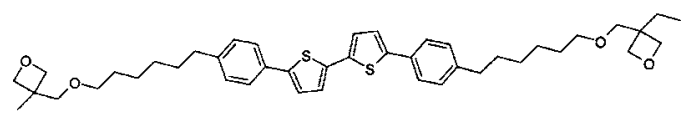

(b)

FIG. 1. (a) Molecular structure of bis(heptylphenyl)-bithiophene (PTTP). (b) Molecular structure of bis(heptylphenyl)-bithiophene-oxetane (PTTP-oxetane). 


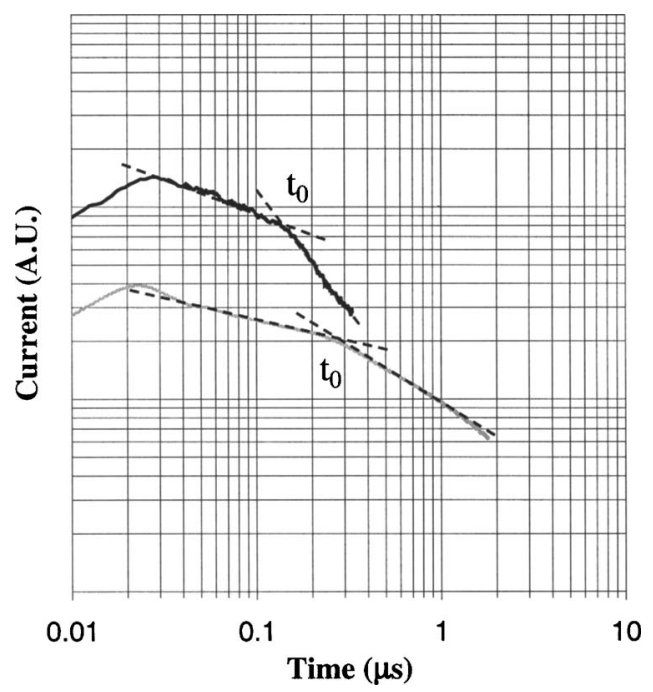

FIG. 2. Electron (upper curve) and hole (lower curve) photocurrents across a $4.9 \mu \mathrm{m}$ sample of PTTP in the smectic phase $\left(E=4 \times 10^{4} \mathrm{~V} \mathrm{~cm}^{-1}\right)$. The dashed lines are included as a guide for the eyes for the location of the transit time, $t_{0}$.

field. The resulting field independent mobility, $\mu_{\text {hole }}$ $=0.016 \mathrm{~cm}^{2} \mathrm{~V}^{-1} \mathrm{~s}^{-1}$, is calculated using $\mu=d^{2} / V t_{0}$ and the inflection point time, $t_{0}$, is used (strictly the arrival time of the fastest carriers) rather than the time to half plateau height (the average arrival time), in order to make comparisons possible with the dispersive electron results, where there is no plateau present. Two electron photocurrents are shown in Fig. 4. They both show a decrease over time before the inflection point, typical of dispersive transport, and the transit time again scales correctly with electric field. The calculated electron mobility is field dependent, as expected for dispersive transport, typically $\mu_{\text {electron }}=0.028 \mathrm{~cm}^{2} \mathrm{~V}^{-1} \mathrm{~s}^{-1}$ at $4 \times 10^{4} \mathrm{~V} \mathrm{~cm}^{-1}$.

Both the hole and electron mobilities measured in this study are remarkable for this kind of cross-linked system. They exceed those measured in cross-linked networks from nematic mesogens $\left(\sim 10^{-4} \mathrm{~cm}^{2} \mathrm{~V}^{-1} \mathrm{~s}^{-1}\right),{ }^{4}$ those measured in smectic mesogens dispersed in a host network

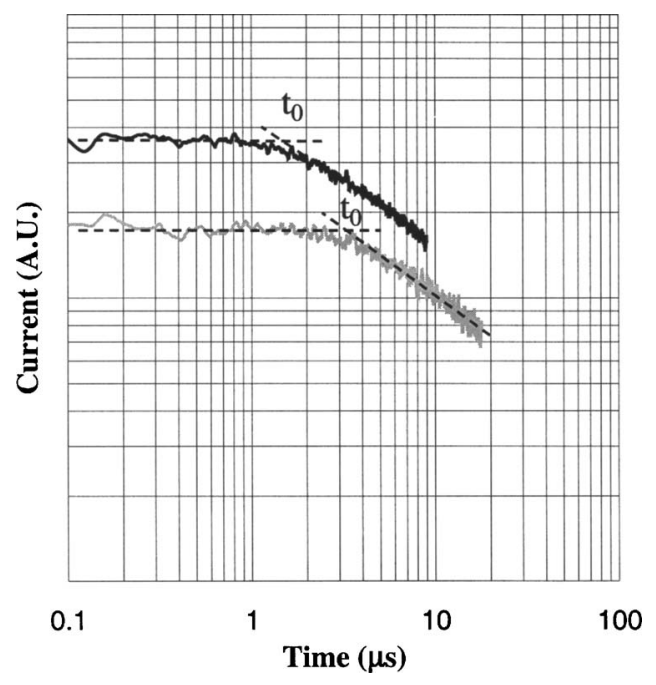

FIG. 3. Hole photocurrent transients across a $10 \mu \mathrm{m}$ film of cross-linked PTTP-oxetane ( $40 \mathrm{~V}$ bias upper curve, $20 \mathrm{~V}$ bias lower curve) at $15^{\circ} \mathrm{C}$. The dashed lines are included as a guide for the eyes for the location of the transit time, $t_{0}$.

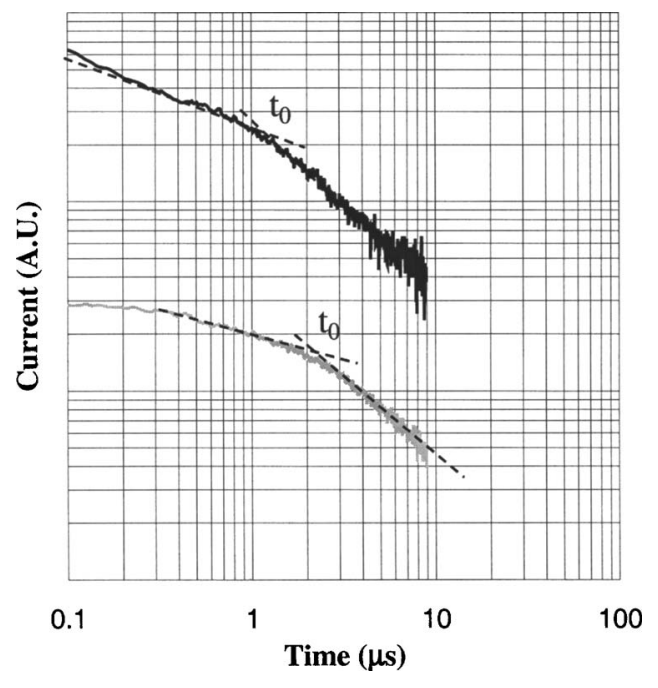

FIG. 4. Electron photocurrent transients across a $10 \mu \mathrm{m}$ film of cross-linked PTTP-oxetane (40 V bias upper curve, $25 \mathrm{~V}$ bias lower curve) at $15{ }^{\circ} \mathrm{C}$. The dashed lines are included as a guide for the eyes for the location of the transit time, $t_{0}$.

$\left(\sim 10^{-3} \mathrm{~cm}^{2} \mathrm{~V}^{-1} \mathrm{~s}^{-1}\right),{ }^{5}$ and those previously reported for cross linked films by the authors. ${ }^{6}$ Although these values are not as large as those for single-molecular crystals, ${ }^{7}$ or those in nonreactive liquid crystal smectic mesophases, ${ }^{8}$ or indeed columnar mesophases, ${ }^{9}$ the formation of a solid, stable, insoluble, and semiconducting layer, will allow easy incorporation of these materials in actual device structures (fieldeffect transistor, organic light-emitting device), in much the same manner as the polymeric materials are currently used. The mobility data for the various materials is summarized in Table I.

An overall consideration of the charge transport in the three systems presented here (PTTP, cross linked PTTPoxetane, and nonpolymerised PTTP-oxetane) leads to the following observations. Both holes and electrons in smectic PTTP display dispersive behavior (Fig. 2). This is unlike the nondispersive transport observed in many other smectic systems (e.g., Refs. 8 and 5) and most probably an impurity effect. The transport in nonpolymerized smectic PTTP-

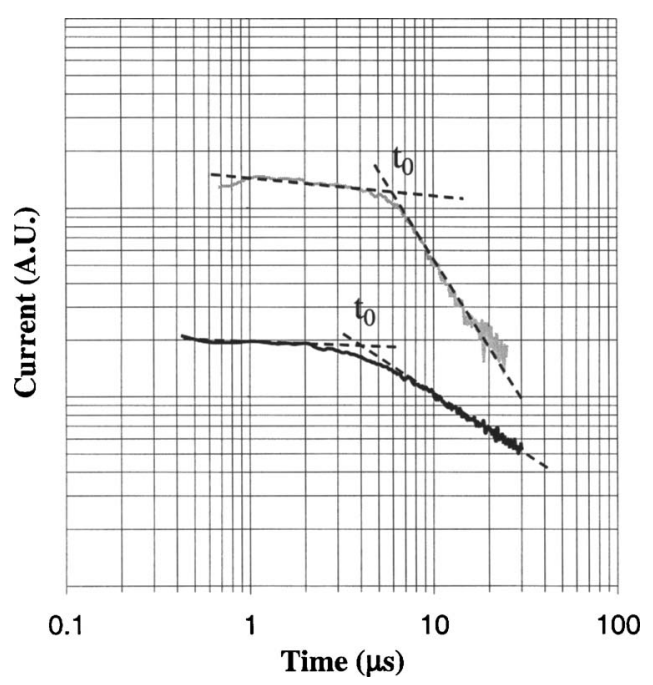

FIG. 5. Hole (upper curve) and electron (lower curve) photocurrents across a $10 \mu \mathrm{m}$ sample of nonpolymerized PTTP-oxetane at $140{ }^{\circ} \mathrm{C}$ (Smectic B phase, $20 \mathrm{~V}$ bias). 
TABLE I. Summary of material parameters.

\begin{tabular}{|c|c|c|c|}
\hline Material & $\mu_{\text {hole }}\left(\mathrm{cm}^{2} \mathrm{~V}^{-1} \mathrm{~s}^{-1}\right)$ & $\mu_{\text {electron }}\left(\mathrm{cm}^{2} \mathrm{~V}^{-1} \mathrm{~s}^{-1}\right)$ & Phase behavior \\
\hline PTTP & $0.044\left(\right.$ at $\left.175^{\circ} \mathrm{C}\right)$ & 0.07 (at $\left.175^{\circ} \mathrm{C}\right)$ & K-140-SmG-225-SmC-229-SmA-234-I \\
\hline PTTP-oxetane & $0.016\left(\right.$ at $\left.15^{\circ} \mathrm{C}\right)$ & $0.028\left(\right.$ at $\left.15^{\circ} \mathrm{C}\right)$ & \\
\hline Cross linked & $0.033\left(\right.$ at $\left.140^{\circ} \mathrm{C}\right)$ & $0.024\left(\right.$ at $\left.140{ }^{\circ} \mathrm{C}\right)$ & \\
\hline PTTP-oxetane nonpolymerized & $0.01\left(\right.$ at $\left.140{ }^{\circ} \mathrm{C}\right)$ & $0.012\left(\right.$ at $\left.140{ }^{\circ} \mathrm{C}\right)$ & K-83-SmG-93-SmB-144-I \\
\hline
\end{tabular}

oxetane appears to be nondispersive for both electrons and holes (both photocurrents display a plateau region, albeit a narrow one in the case of electrons, see Fig. 5), in the absence of photoinitiator, and in agreement with other reported systems. This is in contrast to the cross-linked sample results shown in Fig. 4, where the electron photocurrent becomes dispersive. Any remaining photoinitiator appears to have a trapping effect in the case of electrons, but not in the case of holes, whose behavior remains nondispersive (Fig. 3). The photoinitiator concentration, and its effects on the transport and cross-linking, are the subject of ongoing investigation.

In terms of carrier mobility, there is a small drop between the nonreactive liquid crystal PTTP and the reactive mesogen PTTP-oxetane (both for the cross linked and nonpolymerized case). This is not due to the presence of the photoinitiator, as the nonpolymerised PTTP-oxetane without initiator yields mobilities in agreement with the cross-linked film results at the same temperature (see Table I). There even appears to be some mobility improvement postcross-linking, although this could be due to sample to sample variation. The higher mobility in the nonreactive liquid crystal, compared to the RM, is probably due to the higher order smectic phase $(\mathrm{SmG})$ present in the former compared to the latter $(\mathrm{SmB})$. This relative drop between a liquid crystal and a related reactive mesogen is not surprising and has been previously measured in a variety of systems, ${ }^{10,6}$ although in many cases the drop is of a factor of 10 . This will not be discussed further at present, as the results presented here are a small unoptimized, part of a broader systematic study, involving the variation of both core and reactive end groups in liquid crystals and reactive mesogens, currently being conducted.

The authors wish to thank Dr. K. S. Whitehead (Imperial) for useful discussions on reactive mesogens, Professor J. Goodby and Dr. S. Cowling (York) for the identification of the material mesophases and the EPSRC for financial support of this research.

${ }^{1}$ M. Funahashi and J.-I. Hanna, Appl. Phys. Lett. 71, 602 (1997).

${ }^{2}$ P. Vlachos, S. M. Kelly, B. Mansoor, and M. O'Neill, Chem. Commun. (Cambridge) 2002, 874.

${ }^{3}$ I. McCulloch, W. Zhang, M. Heeney, C. Bailey, M. Giles, D. Graham, M. Shkunov, D. Sparrowe, and S. Tierney, J. Mater. Chem. 13, 2436 (2003).

${ }^{4}$ S. R. Farrar, A. E. A. Contoret, M. O’Neill, J. E. Nicholls, G. J. Richards, and S. M. Kelly, Phys. Rev. B 66, 125107 (2002).

${ }^{5}$ N. Yoshimoto and J.-I. Hanna, J. Mater. Chem. 13, 1004 (2003).

${ }^{6}$ M. Shkunov, W. Zhang, D. Graham, D. Sparrowe, M. Heeney, M. Giles, S. Tierney, C. Bailey, I. McCulloch, and T. Kreouzis, Proc. SPIE 5217, $181(2003)$

${ }^{7}$ V. Podzorov, S. E. Sysoev, E. Loginova, V. M. Pudalov, and M. E. Gershenson, Appl. Phys. Lett. 83, 17 (2003); 83, 3504 (2003).

${ }^{8}$ M. Funahashi and J.-I. Hanna, Adv. Mater. (Weinheim, Ger.) 17, 594 (2005).

${ }^{9}$ D. Adam, P. Schuhmacher, J. Simmerer, L. Haussling, K. Siemensmeyer, K. H. Etzbach, H. Ringsdorf, and D. Haarer, Nature (London) 371, 141 (1994).

${ }^{10}$ R. J. Baldwin (private communication). 